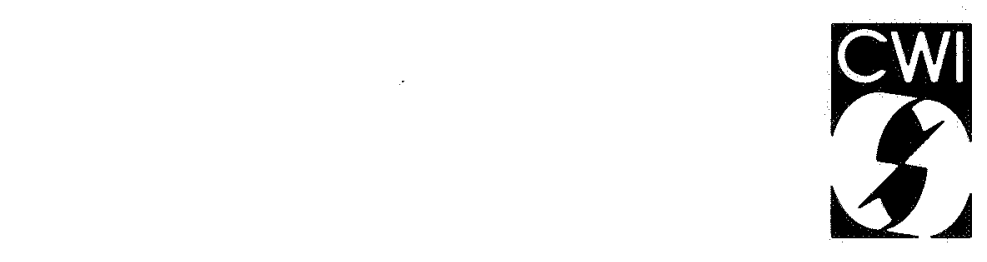

Centrum voor Wiskunde en Informatica Centre for Mathematics and Computer Science

J.B.T.M. Roerdink, H.J.A.M. Heijmans

Mathematical morphology for structures without translation symmetry 
The Centre for Mathematics and Computer Science is a research institute of the Stichting Mathematisch Centrum, which was founded on February 11, 1946, as a nonprofit institution aiming at the promotion of mathematics, computer science, and their applications. It is sponsored by the Dutch Government through the Netherlands Organization for the Advancement of Pure Research (Z.W.O.).

Copyright $\odot$ Stichting Mathematisch Centrum, Amsterdam 


\title{
Mathematical Morphology for Structures \\ without Translation Symmetry
}

\author{
J.B.T.M. Roerdink, H.J.A.M. Heijmans \\ Centre for Mathematics and Computer Science \\ P.O. Box 4079, 1009 AB Amsterdam, The Netherlands
}

\begin{abstract}
Euclidean mathematical morphology is based on the use of translation-invariant transformations of the Boolean lattice of all subsets of the Euclidean space. We discuss how the theory can be generalized to arbitrary complete atomic Boolean lattices with a commutative group structure on the set of atoms, and give some simple examples.
\end{abstract}

1980 Mathematics Subject Classification: 68U10, 06E99.

Key Words \& Phrases: mathematical morphology, complete Boolean lattices, translation invariance.

Note: Contribution to the IEEE-ASSP \& EURASIP Fitth Workshop on Multidimensional Signal Processing,

Sept. 14-16, 1987, Noordwijkerhout, The Netherlands.

\section{INTRODUCTION}

Mathematical morphology on Euclidean spaces is by now a well developed branch of image analysis $[1,2]$. The basic ingredients of the theory are a number of elementary image-to-image transformations (dilation, erosion, hit or miss transform), from which other transformations can be derived(thinning, thickening,...). An important property of these transformations is that they are translation-invariant, i.e. they are adapted to the translation symmetry of the underlying Euclidean space.

For certain applications the use of translation-invariant transformations is not appropriate in view of an internal structure which does not possess translation symmetry. As an example consider Fig. 1, which is a photograph of the trees in a forest, taken by putting the camera at ground level and aiming towards the sky. Such photographs are used to measure the amount of sunshine in the woods. It is clear that in this case we need transformations adapted to the symmetries of this polar structure, i.e. rotations about the intrinsic origin (the projection point of the zenith) and scalar multiplication with respect to the origin. As another example where translation symmetry is broken we mention the pictures taken by weather satellites of large portions of the earth. At this scale the curvature of the earth is no longer negligible and a "spherical morphology" would be in order. 


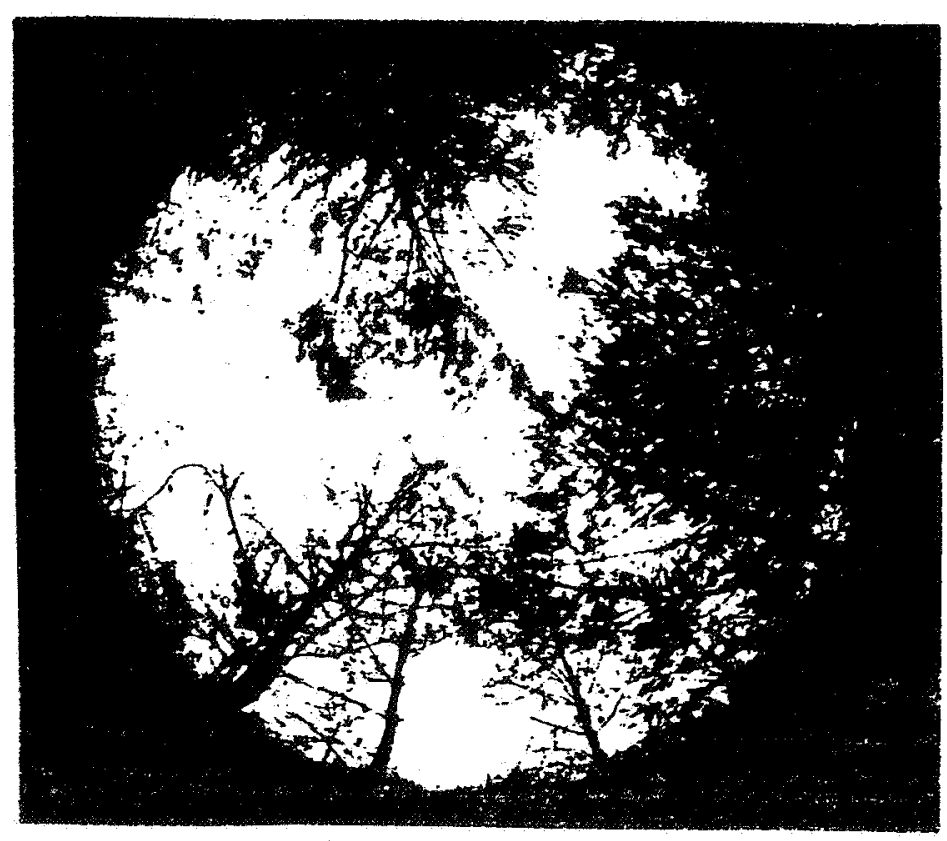

FIGURE 1. Photograph of the amount of sunshine in a forest (from [1], p.17).

\section{BASIC ELEMENTS OF EUCLIDEAN MORPHOLOGY}

Let $E$ be the Euclidean space $\mathbb{R}^{n}$ or the discrete grid $\mathbb{Z}^{n}$. By $P(E)$ we denote the space of all subsets of $E$. A binary image can be represented as a subset $X$ of $E$. If $X \subset E$ and $h \in E$, then $X_{h}$ is the set $X$ translated along the vector $h$ :

$$
X_{h}=\{x+h: x \in X\} \text {. }
$$

On the subsets of $E$ we define the following two elementary algebraic operations (we follow the notation of [1]):

$$
\begin{array}{rll}
\text { Minkowski addition: } & X \oplus A=\cup\left\{X_{a}: a \in A\right\} & X, A \subset E \\
\text { Minkowski subtraction: } & X \ominus A=\cap\left\{X_{a}: a \in A\right\} & X, A \subset E .
\end{array}
$$

The dilation and erosion of a set $X$ by a so-called structuring element $A$ consist of all points $h \in E$ such that the translated set $A_{h}$ hits $X$ or is contained in $X$, respectively. In formulas:

$$
\begin{aligned}
& \text { Dilation: } X \oplus \stackrel{\vee}{A}=\left\{h \in E: A_{h} \cap X \neq \varnothing\right\}=\cup\left\{X_{-a}: a \in A\right\} \\
& \text { Erosion: } X \ominus \stackrel{\vee}{ }=\left\{h \in E: A_{h} \subset X\right\}=\cap\left\{X_{-a}: a \in A\right\} \text {. }
\end{aligned}
$$

Here $A=\{-a: a \in A\}$ is the reflected set of $A$.

Between dilation and erosion there exists a duality relation with respect to set complementation (denoted by the superscript " $c$ "):

$$
(X \oplus \stackrel{\vee}{A})^{c}=X^{c} \ominus \stackrel{\vee}{A},
$$

i.e., dilating an image gives the same result as eroding the background. See Fig. 2 for some simple examples.

Two characteristic properties of dilation are: 
1. Distributivity w.r.t. union: $\left(\bigcup_{i \in I} X_{i}\right) \oplus \dot{A}=\bigcup_{i \in I}\left(X_{i} \oplus A\right)$

2. Translation invariance: $(X \oplus \dot{A})_{h}=X_{h} \oplus \dot{A}$.

Similar properties hold for erosion with intersection instead of union in Eq. (2.7)

A consequence of the distributivity property is that dilation and erosion are increasing transformations. A transformation $\psi: P(E) \rightarrow P(E)$ is called increasing if:

$$
X \subset Y \Rightarrow \psi(X) \subset \psi(Y), \quad \forall X, Y \subset E .
$$

Other examples of increasing transformations are the closing and the opening by a structuring element $A$, defined by:

$$
\begin{array}{cl}
\text { Closing: } & X^{A}:=(X \oplus \breve{A}) \ominus A \\
\text { Opening: } & X_{A}:=(X \ominus \breve{\vee}) \oplus A .
\end{array}
$$

The opening is the union of all the translates $A_{h}$ of $A$ which are included in the set $X$. The complement of the closing of $X$ is the opening of the complement of $X$. An example is given in Fig. 3.

An important theorem of MATHERON [2] states that any increasing translation-invariant mapping $\psi: P(E) \rightarrow P(E)$ is a union of erosions, or, equivalently, an intersection of dilations.

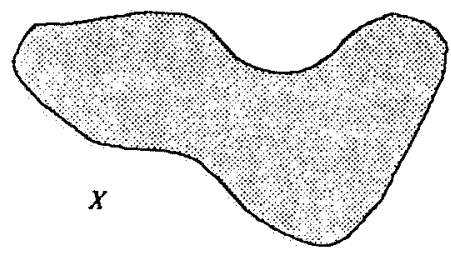

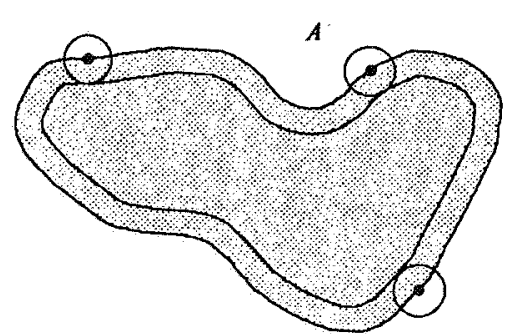

dilation of $X$ by $A$

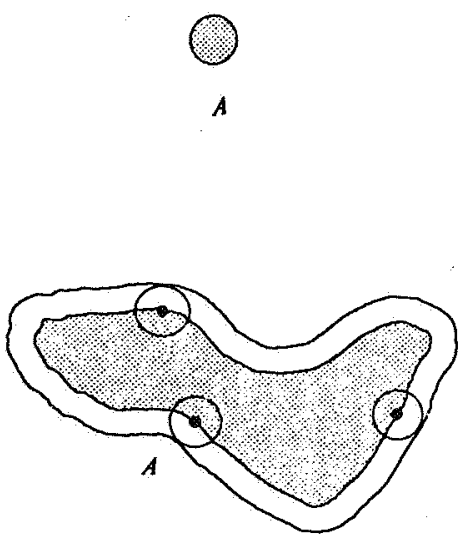

erosion of $X$ by $A$

FIGURE 2a. Dilation and erosion in the continuous case 

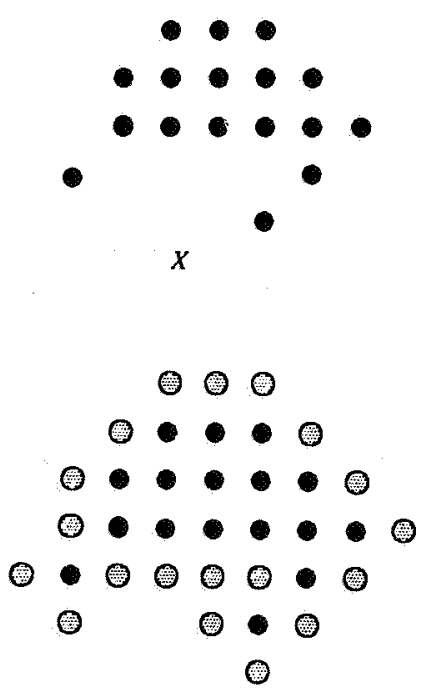

dilation of $X$ by $A$
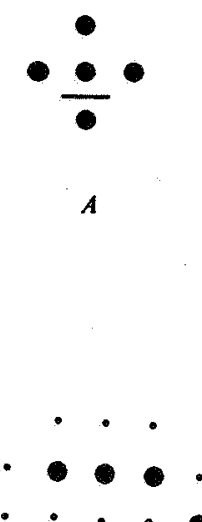

$\cdot \cdot \cdot \bullet$

erosion of $X$ by $A$

FIGURE 2b. Dilation and erosion in the discrete case:

O points which belong to $X \oplus \stackrel{\vee}{A}$ but not to $X$;

- points which belong to $X$;

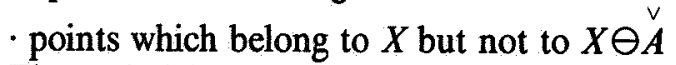

The underlying in $A$ denotes the location of the origin.

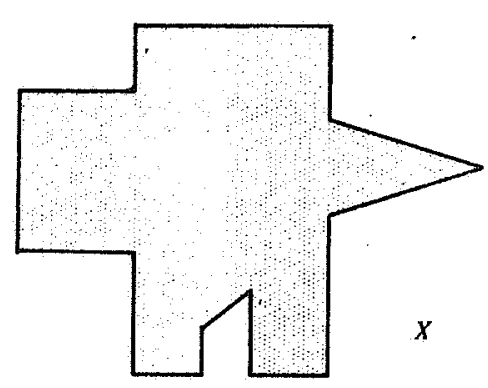

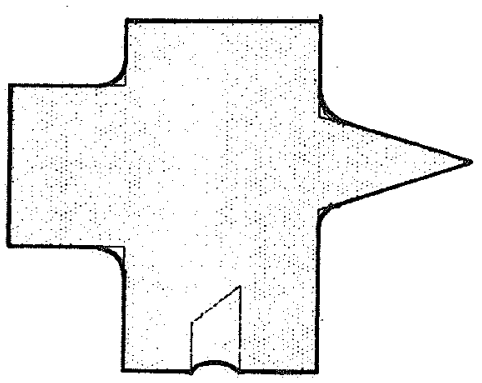

closing of $X$ by $A$

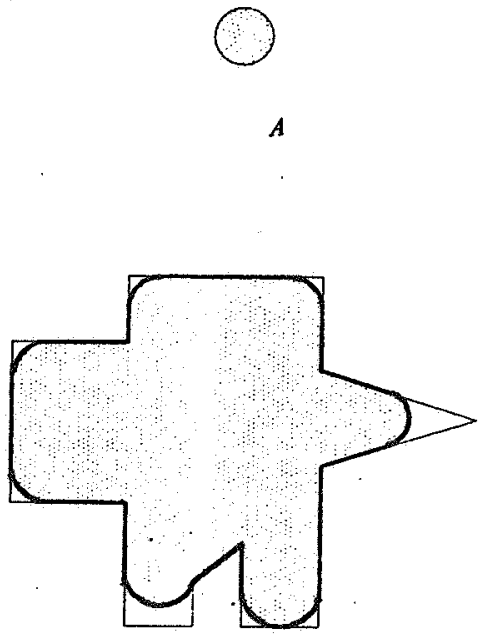

opening of $X$ by $A$

FIGURE 3. Closing and opening in the continuous case 
3. GENERALIZATION OF EUCLIDEAN MORPHOLOGY

An abstract generalization of Euclidean morphology was recently initiated by Heijmans within the setting of lattice theory [3]; see also SERRA [4] and RoNSE [5] for a related approach. Here we confine ourselves to the case of the complete Boolean algebra formed by the space $P(E)$ of subsets of an arbitrary set $E$. The sets $\{x\}$ consisting of one element of $E$ are the so-called atoms of $P(E)$ and $P(E)$ is called an atomic lattice [6].

A generalized definition of dilation and erosion starts from the following observation. The basic algebraic operation in the Minkowski addition and subtraction involves the translation of points of $\mathbb{R}^{n}$ or $\mathbb{Z}^{n}$ along vectors. That is, only part of the vector space structure is actually used, namely the vector additions, which define a commutative group. The set $E$ is called a commutative group if there exists a binary operation $\star: E \times E \rightarrow E$ which has the following properties:

(i) Associativity: $(x \star y) \star z=x \star(y \star z), x, y, z \in E$.

(ii) Commutativity: $x \star y=y \star x, x, y \in E$.

(iii) There is a unit element $\omega \in E$, such that $x \star \omega=x$, any $x \in E$.

(iv) Every $x \in E$ has an inverse $x^{-1}$, with $x \star x^{-1}=\omega$.

This leads one to the following generalization. Let $E$ be an arbitrary commutative group, with group operation *. With every $x \in E$ a mapping $T_{x}: E \rightarrow E$ is associated as follows (we write $T_{x} y$ instead of $\left.T_{x}(y)\right)$ :

$$
T_{x} y=x \star y, \quad y \in E
$$

In the Euclidean case as described in the previous section, $T_{x}$ is the translation along the vector $x$. The family of all such mappings on $E$ is denoted by $G=\left\{T_{x}: x \in E\right\}$. Note that $G$ is itself again a commutative group under composition:

$$
T_{x} T_{y}=T_{x^{\star} y}, \quad x, y \in E .
$$

Just as in the Euclidean case our object space will be the space $P(E)$ of subsets of $E$. The mappings of $G$ work on subsets of $E$ in the obvious way:

$$
T_{x} Y=\{x \star y: y \in Y\}, \quad x \in E, Y \in P(E) .
$$

It is easy to show that for any two subsets $X, Y$ of $E$ and any $z \in E$,

$$
\begin{aligned}
& T_{z}(X \cup Y)=\left(T_{z} X\right) \cup\left(T_{z} Y\right), \\
& T_{z}(X \cap Y)=\left(T_{z} X\right) \cap\left(T_{z} Y\right),
\end{aligned}
$$

i.e. the elements of $G$ preserve unions and intersections. This is expressed in mathematical terminology by saying that $G$ forms a commutative group of automorphisms on $P(E)$, see [3].

Now one sees immediately that all the definitions of section 2 carry over to the general case by substituting the group operation " $\star$ " for the vector addition " + " (in fact, one often uses the symbol " + " for any commutative group operation, but to avoid confusion we have introduced a different notation). So instead of Eq. (2.1), use the following definitions:

$$
X_{h}=\{x \star h: x \in E\}, h \in E ; \quad \stackrel{\vee}{A}=\left\{a^{-1}: a \in E\right\} .
$$

All the standard algebraic properties of Minkowski addition and subtraction, such as

$$
\begin{aligned}
& X \oplus A=A \oplus X \\
& (X \oplus A) \oplus B=X \oplus(A \oplus B) \\
& (X \ominus A) \ominus B=X \ominus(A \oplus B) \\
& (X \cup Y) \oplus A=(X \oplus A) \cup(Y \oplus A) \\
& (X \cap Y) \ominus A=(X \ominus A) \cap(Y \ominus A)
\end{aligned}
$$


remain valid in the general case. Here $X \oplus A$ and $X \ominus A$ are defined as in (2.2) and (2.3), respectively, with (2.1) replaced by (3.1). Instead of "translation invariance", Eq. (2.8), we now have "group invariance" or "G-invariance," and we also sometimes call $X \rightarrow X \oplus A$ and $X \rightarrow X \ominus A$ " $G$-dilations" and " $G$ erosions" to indicate the underlying group structure.

Finally we mention that there also is a generalization of Matheron's Theorem:

THEOREM [3]. All increasing, G-invariant mappings $\psi: P(E) \rightarrow P(E)$ are unions of $G$-erosions and intersections of G-dilations.

Examples are the $G$-openings and $G$-closings.

4. EXAMPLE: A POLAR STRUCTURE

We consider the space $E$ of points in the plane with polar coordinates $(r, \theta)$, where $r$ is the radial distance from the origin $O$ and $\theta$ is the angle with the positive $x$-axis. In the continuous case we take $r>0$ and $\theta \in[0,2 \pi)$, whereas in the discrete case $r=\cdots, a^{-2}, a^{-1}, 1, a, a^{2}, \ldots$, with $a \in \mathbb{R}$, and $\theta=0,2 \pi / m, 4 \pi / m, \ldots, 2 \pi(m-1) / m, m$ a positive integer (see Fig. 4). The group structure of $E$ is defined by the following rule:

$$
\left(r_{1}, \theta_{1}\right) \star\left(r_{2}, \theta_{2}\right)=\left(r_{1} r_{2}, \theta_{1}+\theta_{2}\right) .
$$

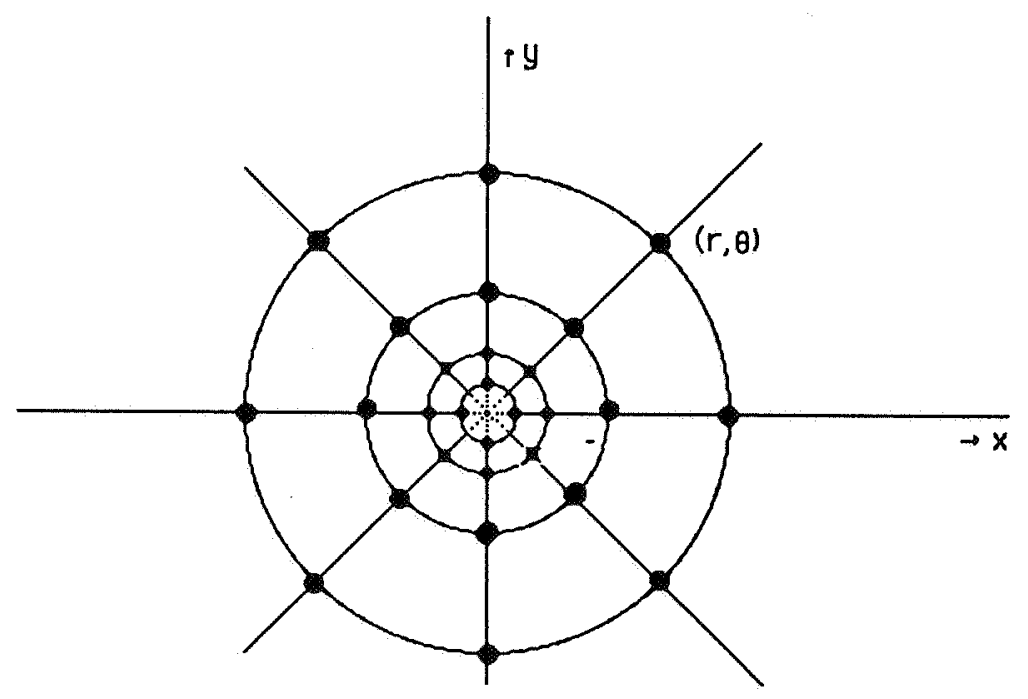

FIGURE 4. Polar space in the discrete case.

So radii are multiplied and angles added, as when multiplying complex numbers. It is easy to check that the set $E$ with the operation * forms a commutative group. The identity element of the group is the point $(1,0)$ and the inverse of $(r, \theta)$ is $\left(r^{-1},-\theta\right)$. Note also that every transformation of the group can be written as a rotation followed by a scalar multiplication, and vice versa:

$$
(r, \theta)=(r, 0) \star(1, \theta)=(1, \theta) \star(r, 0) .
$$

Let us consider transformations based on the group $E$ where the structuring element is a disc $D$ of 
radius $\delta<1$ centered at the identity element $(1,0)$. After scalar multiplication by $(r, 0)$ this becomes a disc of radius $r . \delta$ centered at $(r, 0)$. Rotations only change the center of the disc, not its radius. The Minkowski addition of $D$ and a set $X$ therefore has the form depicted in Fig. 5. To obtain $X \oplus D$, "move" the disc $D$ to all points of $X$, taking into account the proper adjustment of its radius as the radial distance to the origin changes. The union of all the discs $\left\{D_{x}\right\}$ gives $X \oplus D$. To obtain the Minkowski subtraction of $X$ and $D$, take the intersection of all the sets $\left\{X_{d}: d \in D\right\}$, or compute $X^{c} \oplus D$ as above and take the complement.

Note that the reflected disc $\stackrel{V}{D}$ is again a disc: this follows from the fact that the reflection operation corresponds in this case to the fractional linear transformation $z \rightarrow z^{-1}$, where $z=r e^{i \theta}$, which carries circles into circles. The radius of $\stackrel{v}{D}$ is $\delta\left(1-\delta^{2}\right)^{-1}$ and its center is $\left(\left(1-\delta^{2}\right)^{-1}, 0\right)$.

To give an illustration of how the underlying group structure affects the outcome of morphological transformations, we consider the opening by a disc $D$ of radius $\delta$ centered at $(1,0)$. The set $X$ to be opened consists of a number of discs, some of which have the same size as $D$, whereas the remaining discs are smaller than $D$, see Fig. 6a. In Fig. $6 \mathrm{~b}$ the result is presented for the case where the Euclidean translations form the underlying group: all the small discs have disappeared since the disc $D$ does not fit into them. The result for an opening using the rotation-multiplication group is displayed in Fig. 6c. Here the outcome is totally different due to the fact that the radius of the structuring element $D_{x}$ depends on its center $x$ : a disc of radius $R$ centered at $(r, \theta)$ now disappears under the opening if $R<r \delta$.

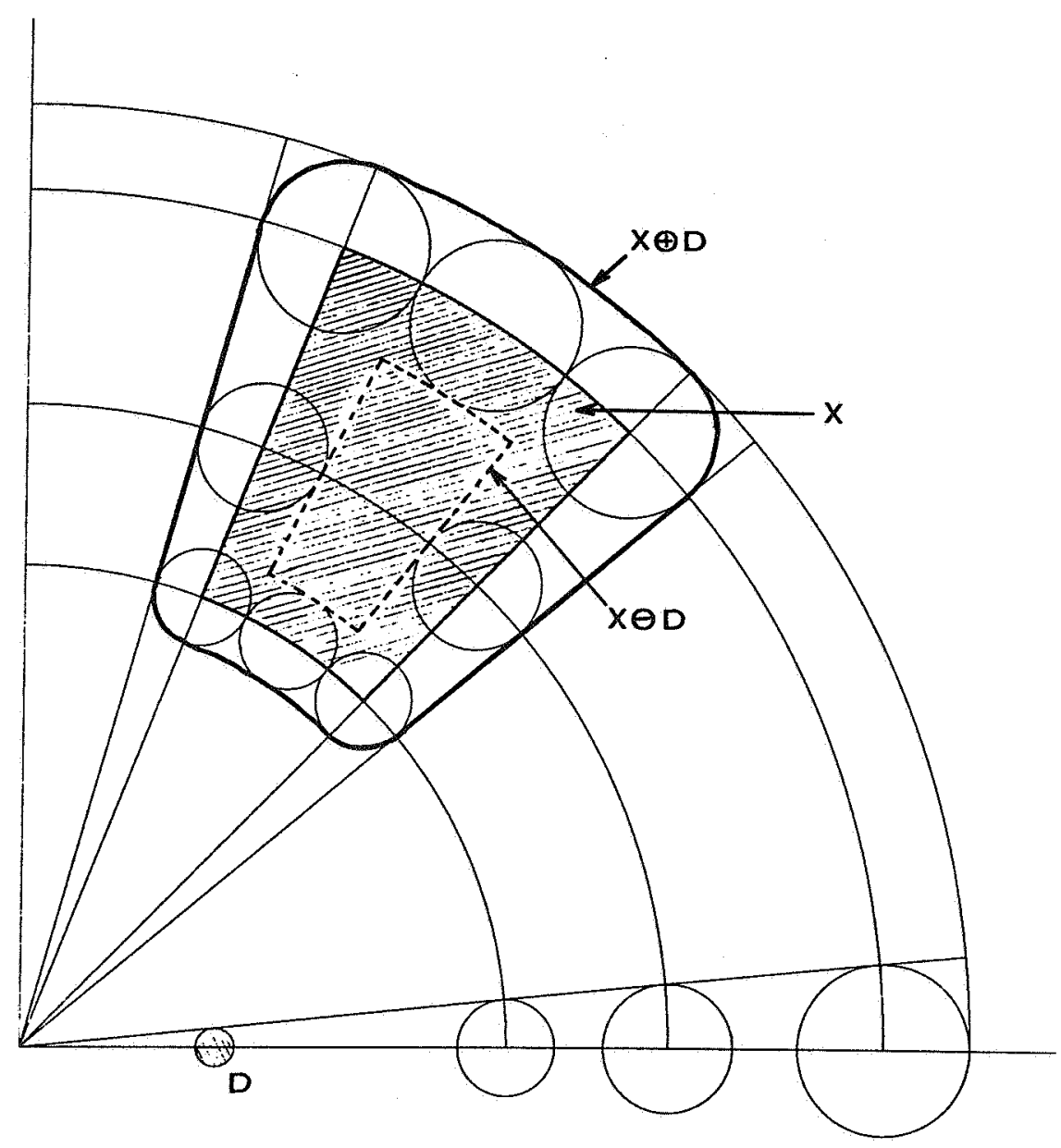

FIGURE 5. Minkowski addition and subtraction of a set $X$ (hatched area) by a disc $D$. The interior - of the solid curve is $X \oplus D$, the interior of the broken curve is $X \ominus D$. 

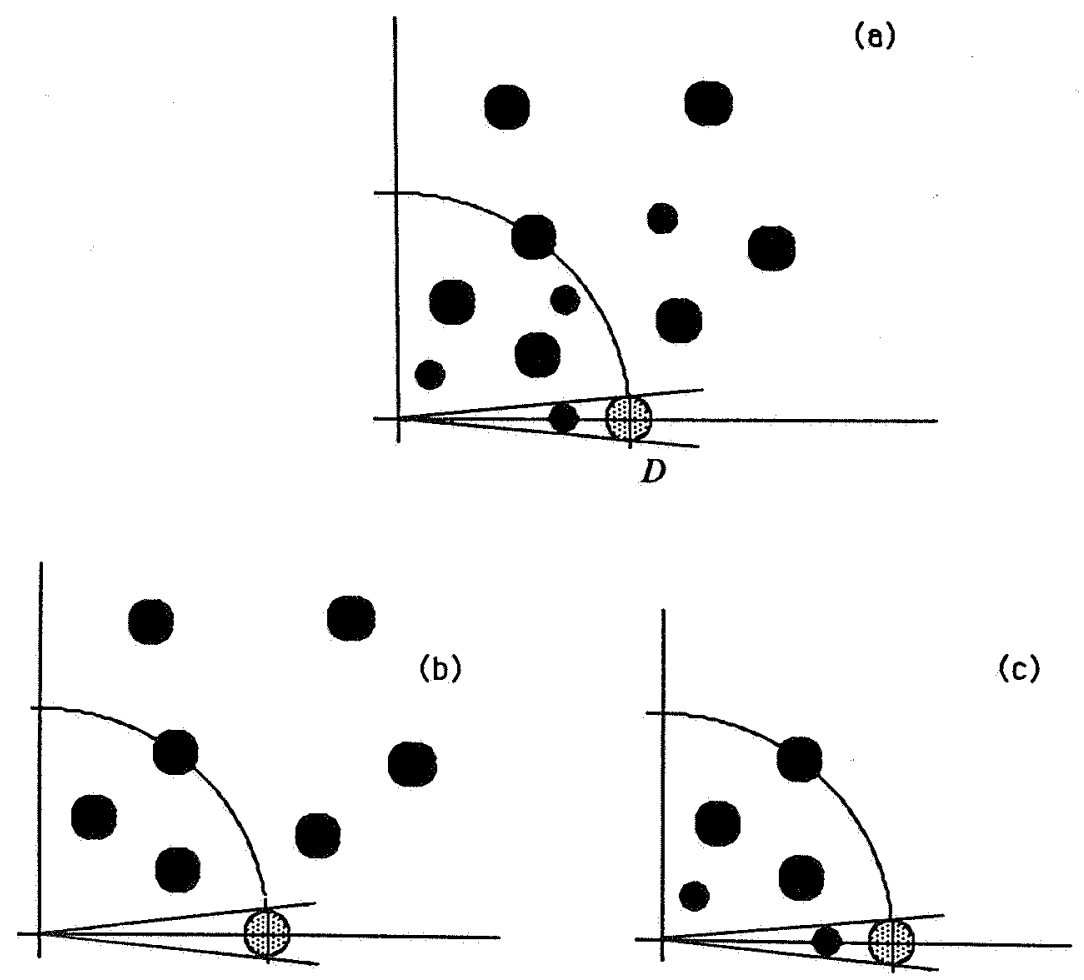

FIGURE 6. (a) the set $X$ (black discs) and the structuring element $D$ (grey).

(b) opening $X_{D}$ with $E=$ translation group.

(c) opening $X_{D}$ with $E=$ rotation-multiplication group.

\section{REFERENCES}

[1] J. SERRA (1982). Image Analysis and Mathematical Morphology, Acad. Press, New York.

[2] A. Matheron (1975). Random sets and integral geometry, Wiley, New York.

[3] H.J.A.M. HeIJMans (1987). Mathematical Morphology: an algebraic approach, CWI Report AM-R8707.

[4] J. SerRA, Advances in Mathematical Morphology, Acad. Press, New York, to appear.

[5] C. RoNSE (1986). A general lattice framework for Morphological operations on pictures, working document, Philips Research Laboratory, Brussels.

[6] G. BIRKHOFF (1967). Lattice Theory (3 rd ed.), AMS, Providence. 